\title{
Enhancing the electrical and physical nature of high- voltage XLPE cable dielectric using different nanoparticles
}

\author{
Abdelrahman Said ${ }^{1, *}$ (D), M. A. Abd-Allah ${ }^{1}$, Amira G. Nawar $^{2}$, Alaa E. Elsayed ${ }^{3}$, and \\ Samir Kamel ${ }^{4}$ \\ ${ }^{1}$ Electrical Engineering Department, Shoubra Faculty of Engineering, Benha University, Cairo, Egypt \\ ${ }^{2}$ Higher Institute for Engineering and Modern Technology, Marg, Cairo, Egypt \\ ${ }^{3}$ Polymer and Pigment Department, National Research Centre, 33 El-Bohouth. Dokki, Giza 12622, Egypt \\ ${ }^{4}$ Cellulose and Paper Department, National Research Centre, 33 El-Bohouth St. Dokki, Giza 12622, Egypt
}

Received: 23 December 2021

Accepted: 28 January 2022

Published online:

18 February 2022

(C) The Author(s) 2022

\begin{abstract}
The goal of this study is to see how different chemically modified nanoparticles affect the performance and characteristics of commercial cross-linked polyethylene (XLPE) as a polymeric insulator used in insulating power cables and to compare their properties in order to figure out what factors are most important in improving the XLPE properties. Silicon dioxide or silica, titanium dioxide, and zinc oxide nanoparticle are used in this study. Nanoparticles (NPs) amino silane surface modification was carried out to decrease nanoparticle aggregation and improve compatibility with the polymer matrix. The melt blending process was used to synthesize and develop XLPE nano-composites on an industrial scale with varying nanoparticle loading ratios $(0.5,2.0,3.5$, and $5.0 \mathrm{wt} \%)$. The morphology and size of all functionalized nanoparticles were explained. The morphology of the produced nano-composites and particle dispersion in the XLPE polymer matrix were studied using X-ray diffraction and field emission scanning electron microscopy. All samples' thermal, electrical, and mechanical properties are evaluated. The result shows the optimum values of melting temperature for functionalized XLPE nano-composites and it is seen that the functionalized $\mathrm{XLPE} / \mathrm{TiO}_{2}$ samples have the highest value that increased by $6.85^{\circ} \mathrm{C}$ over $\mathrm{XLPE}$, but the smallest tensile strength and elongation values were observed. Also, the use of silica NPs gives maximum enhancement dielectric properties.
\end{abstract}

Address correspondence to E-mail: abdelrahman.ghoniem@feng.bu.edu.eg

\section{Introduction}

Polymers are used in a wide range in a lot of electrical applications due to their superior dielectric properties. Polyethylene (PE), polyimide (PI), and 
polypropylene (PP) are examples of these polymer materials. However, despite their good insulation properties, practically, there is still an urgent need to develop them to match the power system requirements. Previously, many researchers have worked on the objective of utilizing the concept of using nanomaterial composites [1]. With the emergence of nanoparticles (NPs) in previous few decades, particulate composites have gotten a lot of interest. NPs have an important role in increasing the electrical, mechanical, and thermal properties of nano-composites at low concentrations while maintaining other features [2]. In recent years, many authors presented in their studies the development of polymer materials using the composite concept to improve the all properties and increase the lifetime and reliability of the insulators [3-12].

The lack of compatibility among the organic polymer, like PE and PI, and the inorganic nanofiller, like silica, is a significant problem. As a result, an obvious tendency for NPs aggregation can happen. The solution of this problem lies in NPs surface treatment in two ways: firstly, removing the NPs surface free energy and, secondly, boosting compatibility between inorganic nanofillers and the organic polymer matrix [13]. Nano-composites are found to have advantages over micron-filled polymers in terms of increased degradation resistance and improved thermo-mechanical properties without a drop in dielectric strength when compared to micron-filled polymers [14]. The internal surfaces of nano-filled materials are crucial in determining their properties. NPs have a high surface-area-to-volume ratio, especially, for NPs having size less than $100 \mathrm{~nm}$, which is resulting in nano-composites that have a larger interfacial area (interaction zone) than micro-composites. This zone causes a considerable mass of polymer to surround the particle which is affected by the particle surface state and has properties that differ from neat polymer [14].

In this study, authors investigated the effect of incorporating different types of functionalized silicon dioxide $\left(\mathrm{SiO}_{2}\right)$, titanium dioxide $\left(\mathrm{TiO}_{2}\right)$, and zinc oxide $(\mathrm{ZnO})$ with $0.5,2.0,3.5$, and $5.0 \mathrm{wt} \%$ into crosslinked polyethylene (XLPE) polymer used in highvoltage underground cable, a single-conductor 2500 $\mathrm{mm}^{2}$ AL-sheathed XLPE cable with the insulation thickness of $27 \mathrm{~mm}$, shown in Fig. 1 [15]. The authors used an industrial method represented in the melt blending method in nano-composites sample preparation and show the nanofillers effect on the statistical characteristics of XLPE. The size of the NPs was carefully measured using transmission electron microscopy (TEM). X-ray diffraction (XRD) and field emission scanning electron microscopy (FE-SEM) were used to characterize the samples of fabricated nano-composited XLPE. Moreover, the dielectric properties such as relative permittivity and loss tangent have been measured. A test cell was also used to determine the dielectric strength in accordance with ASTM D149-09 "Standard Test Method for Dielectric Breakdown Voltage and Dielectric Strength of Solid Electrical Insulating Materials at Commercial Power Frequencies" [16]. Mechanical properties, such as elongation and tensile strength, were also assessed. The results for various types of nano-composites were then compared in a systematic manner.

\section{Methodology}

\subsection{Materials}

CLNA $8141 \mathrm{EHV}$ is a low-density XLPE compound that is used to insulate extra-high-voltage AC and DC power cables. El- Sewedy Electric Co. furnished it. The $\mathrm{SiO}_{2} \mathrm{NPs}$ were made by charging silica gel into a muffle furnace at $\left(900{ }^{\circ} \mathrm{C}\right)$ for (5) $\mathrm{h}$ under atmospheric conditions. The Titanium (IV) oxide (CAS 13463-67-7) NPs with a molecular weight of $(79.870 \mathrm{mg} / \mathrm{mol})$ with a nano-powder purity of 99.5\%, $\mathrm{TiO}_{2} \mathrm{NPs}$ were purchased from SigmaAldrich. Practically, to produce ZnO NPs, $1314 \mathrm{mg}$ of zinc acetate and $480 \mathrm{mg}$ of $\mathrm{NaOH}$ were dissolved in $330 \mathrm{ml}$ ethanol and refluxed at $60{ }^{\circ} \mathrm{C}$ for about $60 \mathrm{~min}$. After the reaction was completed, adding deionized water, the white solid products were centrifuged at $7000 \mathrm{rpm}$ for $5 \mathrm{~min}$ to separate them from the dispersion supernatant, followed by drying in a laboratory electrical oven at $60{ }^{\circ} \mathrm{C}$ [17]. For surface activation, LOBA Chemie's $98 \%$ pure methane-sulfonic acid used. The coupling agent used here is gamma-amino propyltriethoxy silane "amino silane" which has a purity of $99 \%$ and was acquired from Momentive Inc.

\subsection{Nanoparticle functionalization}

The major roles of surface functionalization are as follows: I. To stabilize the nanoparticles within a 
polymer matrix (obtain a good dispersion of the nanoparticles) via chemical bonding, II. To obtain chemical compatibility and thermodynamical between the polymer and incorporated filler and keep the particles separated from each other; and III. To improve the adherence at the interfaces between matrix and particles [17].

Two steps are involved in the surface modification process. The NPs were etched with hydroxyl groups using acid. In step No. 1, "surface activation", at $110^{\circ} \mathrm{C}$ for $4 \mathrm{~h}, 10 \mathrm{~g}$ of a certain type of NPs was mixed with $100 \mathrm{ml}$ of $10 \%$ methane-sulfonic acid. The powder was then collected in a Hitachi centrifuge for $5 \mathrm{~min}$ at $1500 \mathrm{rpm}$, cleaned multiple times with deionized water, and dried for $24 \mathrm{~h}$ at $120^{\circ} \mathrm{C}$ in a vacuum oven.

The activation of NPs was done by using amino silane in step no. 2, "surface modification", where $3000 \mathrm{mg}$ of activated NPs was dispersed in $60 \mathrm{ml}$ of toluene in a round flask for sonication in an Elmasonic S-60H water bath sonicator for $30 \mathrm{~min}$ at $70{ }^{\circ} \mathrm{C}$. Then, at $70{ }^{\circ} \mathrm{C}$, the mixture is agitated for about $2 \mathrm{~h}$ at $500 \mathrm{rpm}$. Dropwise, a 10\% (wt/wt) amino silane solution in toluene was added, and the stirring procedure lasted for $8 \mathrm{~h}$. Finally, the produced functionalized NPs were collected and washed with isopropanol and then dried for $24 \mathrm{~h}$ in a vacuum oven at $120{ }^{\circ} \mathrm{C}$ [17-19].

\subsection{XLPE/nano-composite preparation}

The melt blending approach was adopted to prepare all samples of XLPE/nano-composites in this work. In the El-Sewedy Egy-tech laboratory, this procedure is known as the master batch method. 170,000 $\mathrm{mg}$ of XLPE pellets with various weights of functionalized $\mathrm{NPs}\left(\mathrm{SiO}_{2}, \mathrm{TiO}_{2}\right.$, and $\left.\mathrm{ZnO}\right)$ make up each nanocomposite formulation $(0.5,2,3.5$ and $5 \mathrm{wt} \%)$. The sample dimensions are of $20 \times 20 \mathrm{~cm}$ with 0.105 to $0.23 \mathrm{~cm}$ thicknesses, the process of these sheets' modification is as follows:

After mixing XLPE pellets with NPs at $150{ }^{\circ} \mathrm{C}$ for $10 \mathrm{~min}$ at $75 \mathrm{rpm}$, the mixture was annealed at $60{ }^{\circ} \mathrm{C}$ for $24 \mathrm{~h}$. Then, it was then blended at $140{ }^{\circ} \mathrm{C}$ for $15 \mathrm{~min}$ at $30 \mathrm{rpm}$ in a double rolls mixer (Battaggion, MCC $150 * 300 / \mathrm{R}-\mathrm{o})$. The samples were pressed for 20 min at $150{ }^{\circ} \mathrm{C}$ under 200 bars with a GDB Laboratory hot/cold press (Gibitre instruments Co.). Finally, the samples were boiled for 3-4 h in water and allowed to cool slowly.

\subsection{Characterization of nano-composites}

High-resolution TEM (JEOL.JEM.2100) was used to describe the form and size of $\mathrm{SiO}_{2}, \mathrm{TiO}_{2}$, and $\mathrm{ZnO}$ NPs. At $120 \mathrm{kV}$ acceleration voltage, the images were collected. FE-SEM (Quanta FEG-250, FEI Inc.) was used to examine the surface morphology of nanocomposites. The energy dispersive $\mathrm{X}$-ray analysis (EDX) equipment was connected to the microscope. The accelerating voltage was set in between 10 and $15 \mathrm{kV}$ to capture the images. The XRD patterns of nano-composites were studied using a Diano XRD with a CoK radiation source powered at $45 \mathrm{kV}$ and a Philips XRD (PW 1930 generator, PW 1820 goniometer) with a $\mathrm{CuK}$ radiation source $(\lambda=0.15418 \mathrm{~nm})$ in reflection mode, with a diffraction angle range of $2 \theta$ from $10^{\circ}$ to $80^{\circ}$. The thermal stability of XLPE nano-composites was investigated using a STA 6000 Perkin Elmer Analyzer and a thermogravimetric analysis (TGA, Shimadzu DTG60, Japan) from 25 to $900{ }^{\circ} \mathrm{C}$ at a heating rate of $10^{\circ} \mathrm{C} / \mathrm{min}$ under argon. The dielectric strength, relative permittivity, and loss tangent were all used to describe dielectric qualities. The dielectric parameters of the produced samples, such as loss tangent and permittivity, were measured using a Hioki 3532-50 LCR Hi Tester with a frequency range of $\left(1-1 \times 10^{6} \mathrm{~Hz}\right)$. The dielectric strength is also tested using an ASTM D149-09 test cell, which consists of a sphere-to-sphere setup powered by a $400 \mathrm{~V} / 250 \mathrm{kV}$ test transformer. The measure of mechanical characteristics was done by using ASTM D1708-18 (standard test method for tensile characteristics of plastics by micro-tensile specimens) $[20,21]$ to define the elongation and tensile strength, which were studied at room temperature.

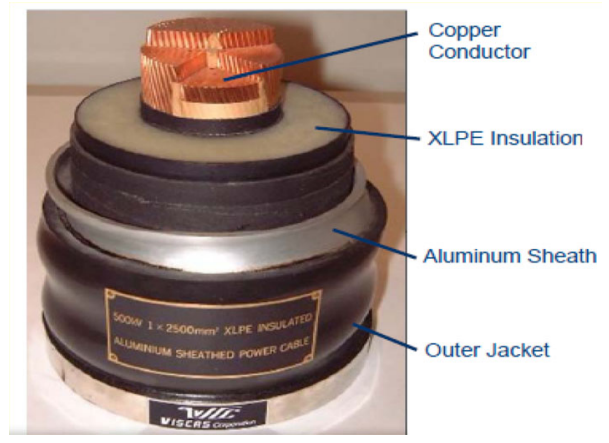

Fig. 1 Cable configurations 


\section{Morphological analyses}

\subsection{Specification of nanoparticles}

The NPs size was accurately measured using TEM. As shown in Fig. 2a-c, the average sizes of $\mathrm{SiO}_{2}, \mathrm{TiO}_{2}$, and $\mathrm{ZnO}$ nanoparticles were about $15 \mathrm{~nm}$, 60-160 nm, and 6-12 nm, respectively.

\subsection{XLPE nano-composites micrographs}

The FESEM micrographs of the XLPE surface without nanoparticles are presented in Fig. 3a. Comparing the FE-SEM micrographs for the surface of XLPE nanocomposites in Fig. 3b-d with neat XLPE in Fig. 3a shows the effects of amino silane surface treatments on the dispersion of the nanoparticles inside the XLPE chains. When amino-functionalized nanoparticles are used, a useful and homogeneous distribution of nanoparticles inside the polymer matrix is seen.

\subsection{Crystallinity of XLPE nano-composites, XRD}

Figure 4 shows the XLPE diffraction pattern in X-rays. An amorphous hallo may be detected, as well as two prominent crystalline peaks at $2 \theta=21.40^{\circ}$ $(d=4.15112 \AA)$ and $2 \theta=24.06^{\circ}(d=3.777 \AA)$, where $d$ is the interplanar distance. Table 1 summarizes the other secondary peaks.

The addition of different NPs forms to XLPE had no effect on the main crystalline peaks at $2 \theta=21.40$ and $24.06^{\circ}$. Small variations in interplanar spacing were observed at a value of $\left(6 \times 10^{-3}-1 \times 10^{-2} \AA\right)$, with an additional peak at $2 \theta=38.1199^{\circ}$ $(d=2.36080 \AA)$ in case of $\mathrm{XLPE} / \mathrm{SiO}_{2}$ functionalized nano-composites, which represents crystallization of $\mathrm{SiO}_{2}$ NPs inside the polymer matrix, and two additional peaks at $2 \theta=27.3837^{\circ}(d=3.25702 \AA)$ and $2 \theta=68.9254^{\circ}(d=1.36127 \AA)$ or $\mathrm{XLPE} / \mathrm{TiO}_{2}$ functionalized nano-composites, respectively, which represents the crystallization of $\mathrm{TiO}_{2} \mathrm{NPs}$. There were no additional peaks found in XLPE/ZnO nanocomposites.
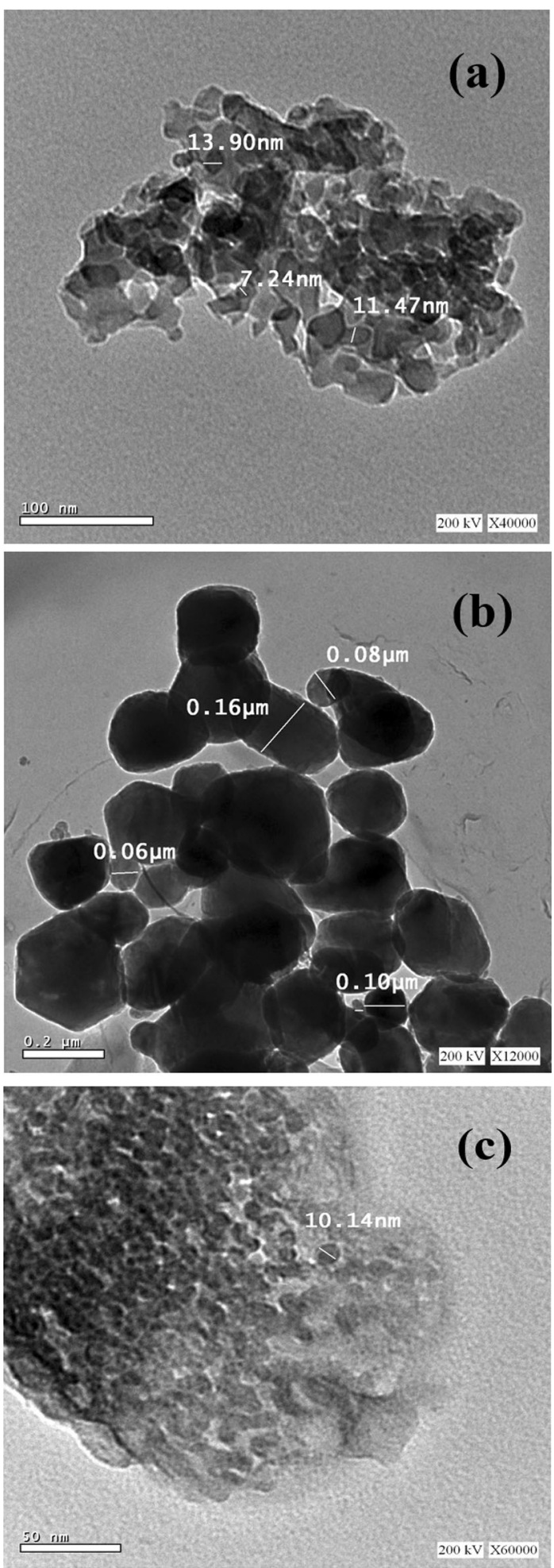

Fig. 2 TEM micrographs of functionalized nanoparticles a $\mathrm{SiO}_{2}$, b $\mathrm{TiO}_{2}$, and c $\mathrm{ZnO}$ 

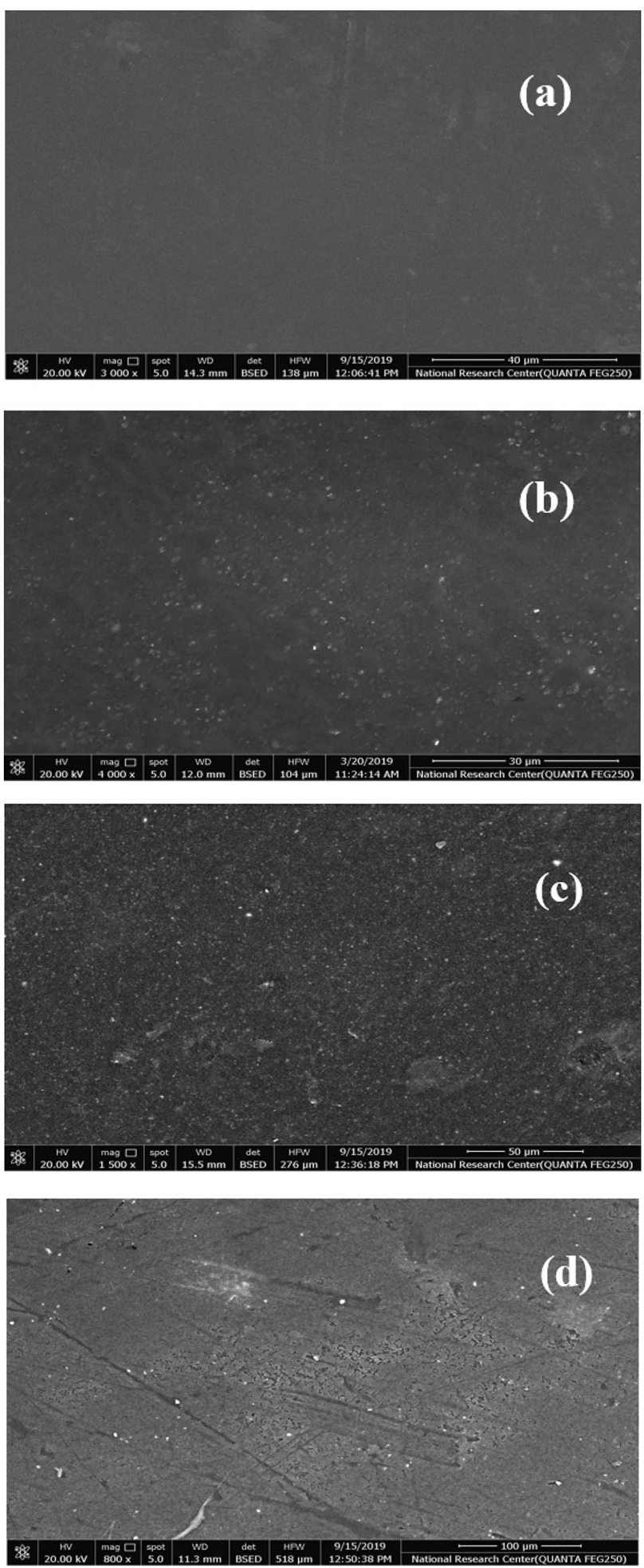

Fig. 3 XLPE and functionalized XLPE nano-composites surface FE-SEM micrographs: a XLPE, b XLPE/SiO ${ }_{2}$, c XLPE/TiO 2 , and d XLPE/ZnO

\section{Results and discussion}

\subsection{Comparison based on thermal characteristics}

Figure 5 shows the optimum breakdown temperatures for XLPE and functionalized XLPE nano-composites. The functionalized $\mathrm{XLPE} / \mathrm{ZnO}$ clearly had the greatest value, increasing by $8.03{ }^{\circ} \mathrm{C}$ above the neat XLPE. This indicates greater thermal stability, as functionalized XLPE/ $\mathrm{TiO}_{2}$ increased by $7.66{ }^{\circ} \mathrm{C}$, followed by $\mathrm{XLPE} / \mathrm{SiO}_{2}$ by $4.32{ }^{\circ} \mathrm{C}$. It was discovered that including the functionalized NPs into the XLPE matrix increased the XLPE thermal stability. The raise in the thermal resistance of the inorganic filler is responsible for this outcome [22-24]. Figure 6 illustrates the optimum glass transition temperatures $(\mathrm{Tg})$ for XLPE and XLPE-functionalized nano-composites, with the functionalized $\mathrm{XLPE} / \mathrm{ZnO}$ having the highest value, increasing by $4.9^{\circ} \mathrm{C}$ over $\mathrm{XLPE}$, followed by functionalized $\mathrm{XLPE} / \mathrm{TiO}_{2}$ increasing by $2.65{ }^{\circ} \mathrm{C}$, and $\mathrm{XLPE} / \mathrm{SiO}_{2}$ increasing by $1.44{ }^{\circ} \mathrm{C}$. Figure 7 shows the optimum values of melting temperature $\left(\mathrm{T}_{\mathrm{m}}\right)$ and it is seen that the functionalized $\mathrm{XLPE} / \mathrm{TiO}_{2}$ has the highest value that increased by $6.85{ }^{\circ} \mathrm{C}$ over neat XLPE followed by functionalized

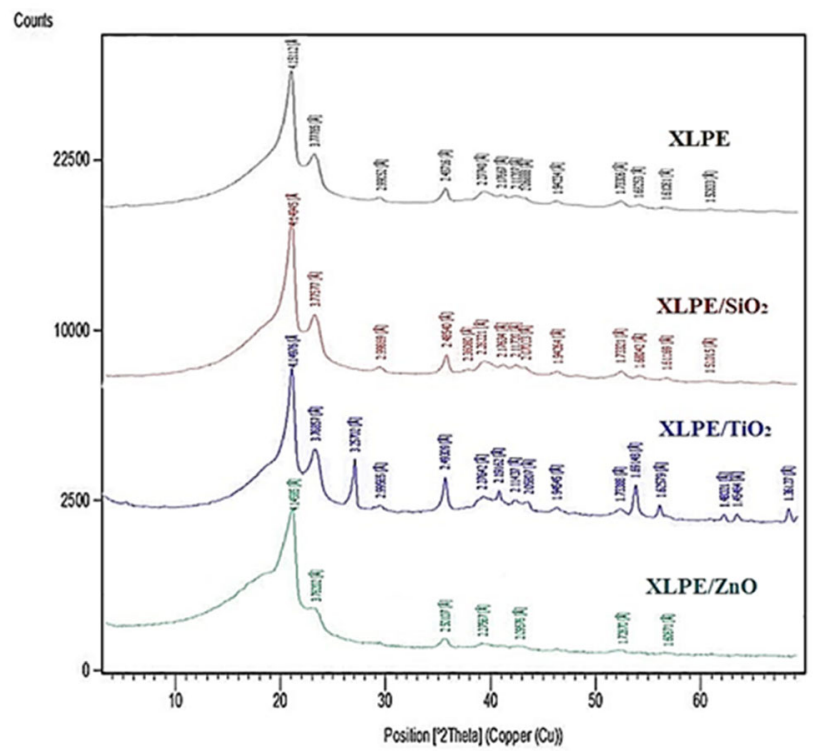

Fig. 4 X-ray diffraction patterns of neat XLPE and functionalized XLPE nano-composites 
Table 1 Small crystalline peaks

\begin{tabular}{ll}
\hline $2 \theta$ & (Interplanar distance) $d(\AA)$ \\
\hline $29.8784^{\circ}$ & 2.99052 \\
$39.573^{\circ}$ & 2.27740 \\
$41.4867^{\circ}$ & 2.17667 \\
$42.8169^{\circ}$ & 2.11207 \\
$43.7564^{\circ}$ & 2.06888 \\
$46.7704^{\circ}$ & 1.94234 \\
$52.8265^{\circ}$ & 1.73306 \\
$54.5422^{\circ}$ & 1.68253 \\
$57.1883^{\circ}$ & 1.61081 \\
$61.4206^{\circ}$ & 1.50833 \\
\hline
\end{tabular}

XLPE/ZnO which increased by $6.46{ }^{\circ} \mathrm{C}$ then XLPE/ $\mathrm{SiO}_{2}$ by $4.97^{\circ} \mathrm{C}$.

\subsection{Comparison based on mechanical characteristics}

The mechanical properties for XLPE and functionalized XLPE nano-composites include tensile strength and elongation that were studied. The mechanical properties were enhanced by incorporating functionalized NPs within the XLPE polymer matrix which decreased the elongation and increased the tensile strength in most samples. This is due to the presence of nano-sized inorganic particles within the XLPE polymer matrix, which enhances the particular surface area accessible for the matrix's interactions. In addition, the restriction of chain mobility caused by the interaction between NPs and the polymer matrix

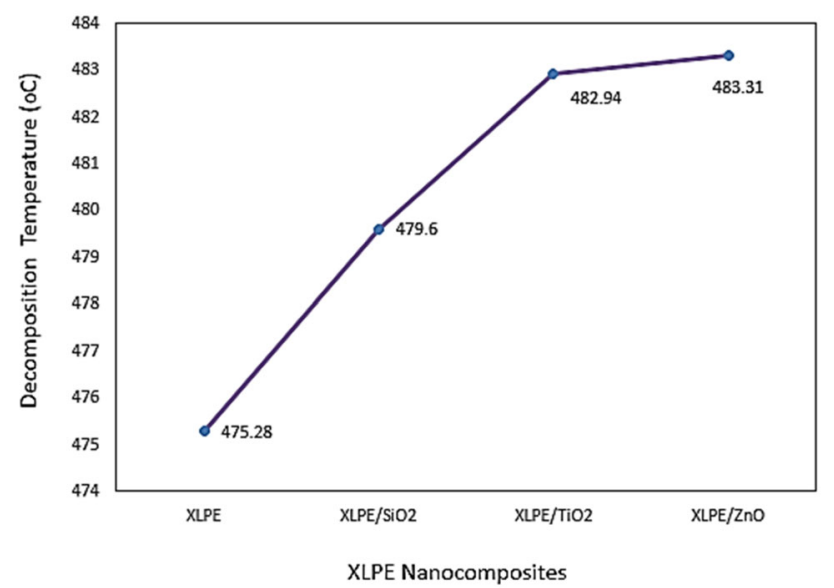

Fig. 5 Decomposition temperatures of XLPE and functionalized XLPE nano-composites

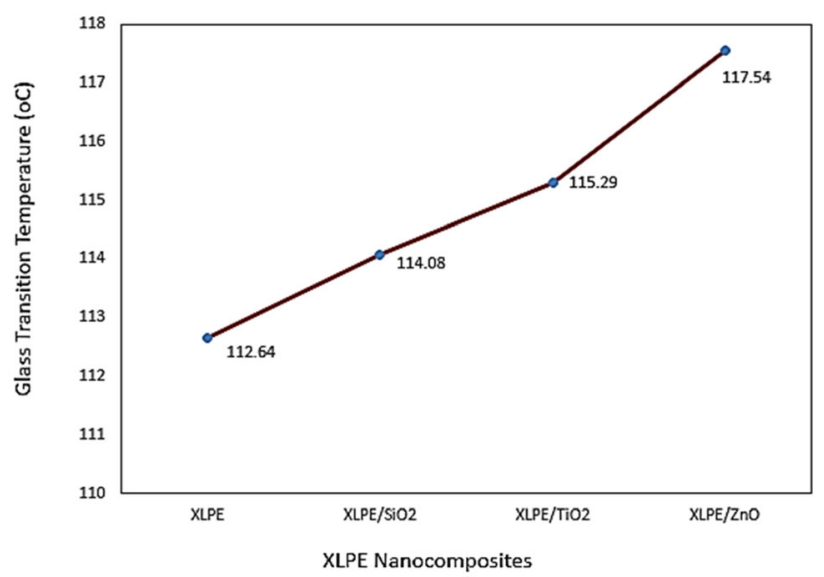

Fig. 6 Glass transition temperature of XLPE and functionalized XLPE nano-composites

may result in elongation reduction with the addition of filler [24].

Figure 8 shows the XLPE and XLPE-functionalized nano-composites tensile strength. It is shown that the smallest tensile strength was observed on XLPE/TiO curve and the highest value was observed at (5.0 wt $\%)$ XLPE $/ \mathrm{SiO}_{2}$ which increased by $46.0 \%$ over that of neat XLPE followed by (3.5 wt \%) functionalized XLPE/ZnO which increased by $43.0 \%$ and then (3.5 wt $\%)$ functionalized $\mathrm{XLPE} / \mathrm{SiO}_{2}$ samples which increased by $41.0 \%$. Figure 9 shows the elongation values of XLPE and functionalized XLPE nanocomposites.

It is clear that the smallest elongation values were observed on functionalized $\mathrm{XLPE} / \mathrm{TiO}_{2}$ curve and the lowest value was at $(0.5 \mathrm{wt} \%)$ functionalized XLPE/ $\mathrm{TiO}_{2}$ which decreased by $45.2 \%$ than neat XLPE,

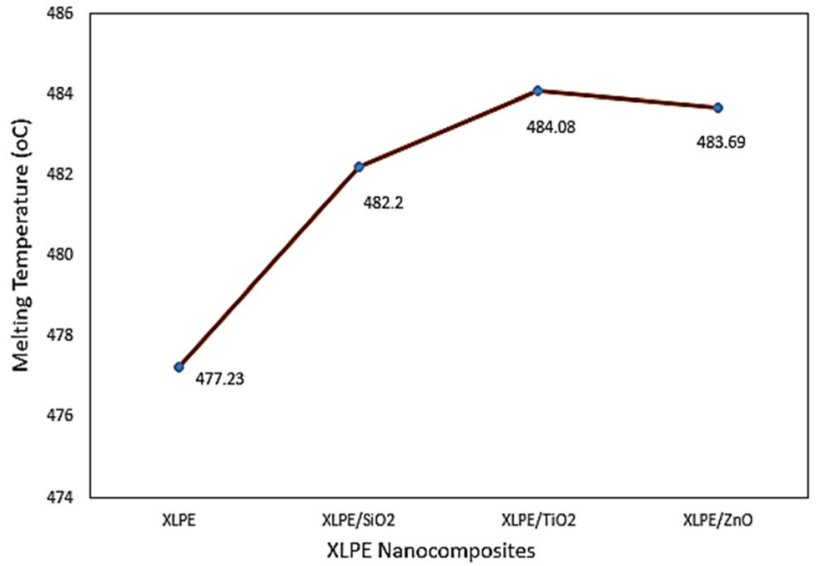

Fig. 7 Melting temperature of XLPE and functionalized XLPE nano-composites 
followed by (0.5 wt \%) functionalized XLPE/ZnO samples which decreased by $40.1 \%$, and then ( $5 \mathrm{wt} \%$ ) functionalized XLPE/ZnO samples which decreased by $34.4 \%$.

\subsection{Comparison based on dielectric characteristics}

Figures 10 and 11 demonstrate the dielectric loss and relative permittivity for XLPE and produced XLPE nano-composite samples at $0.050 \mathrm{kHz}$ for varied $\mathrm{SiO}_{2}, \mathrm{TiO}_{2}$, and $\mathrm{ZnO}$ loadings. Figure 10 shows that the XLPE/ZnO (0.5 wt\%) sample had the greatest increase in relative permittivity, increasing by $8.9 \%$ when compared to neat XLPE. The smallest reduction in relative permittivity was recorded at $\mathrm{XLPE} / \mathrm{SiO}_{2}(2$ wt $\%)$ and XLPE/ZnO (2.0 wt\%), both of which were reduced by $4.1 \%$. It can be remarked that silica NPs provide the greatest increase enhancement in relative permittivity values.

For dielectric loss and in case of $\mathrm{XLPE} / \mathrm{SiO}_{2}$ and $\mathrm{XLPE} / \mathrm{TiO}_{2}$ nano-composites, all samples recorded losses less than neat XLPE, and the maximum enhancement has been observed at $\mathrm{XLPE} / \mathrm{TiO}_{2}(5$ ${ }_{\mathrm{wt}} \%$ ) sample that reduced by $91.6 \%$ compared with neat XLPE as shown in Fig. 11. It is seen that the maximum enhancement in dielectric losses values takes place with using titanium dioxide NPs.

The mean values of the measured AC breakdown voltage (AC BDV) of XLPE and prepared XLPEfunctionalized nano-composite samples at varying $\mathrm{SiO}_{2}, \mathrm{TiO}_{2}$, and $\mathrm{ZnO}$ loadings are shown in Fig. 12. When comparing neat XLPE to XLPE/amino functionalized NPs samples under sphere-sphere field, it

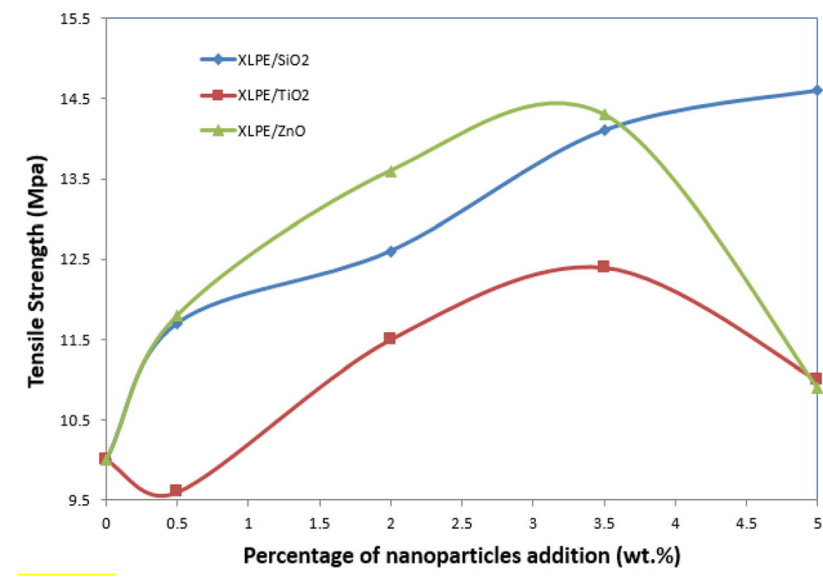

Fig. 8 XLPE and functionalized XLPE nano-composites tensile strength

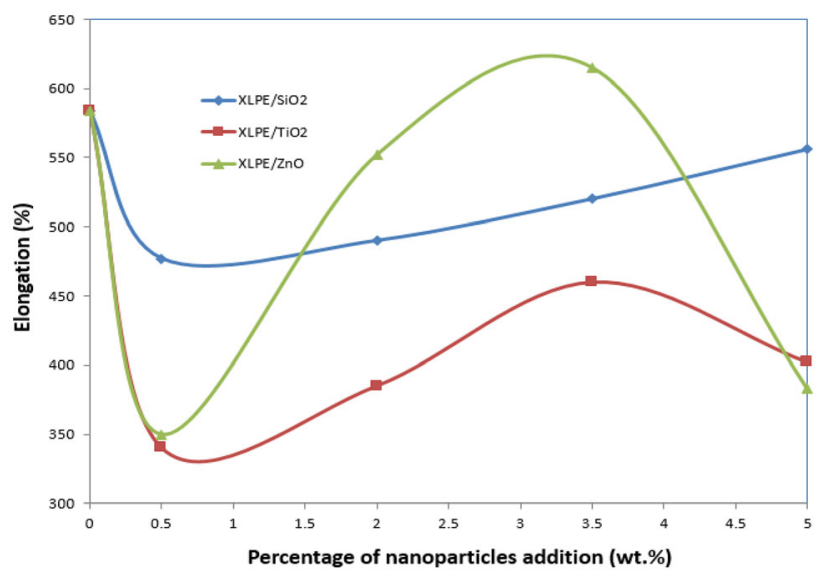

Fig. 9 XLPE and functionalized XLPE nano-composites elongation

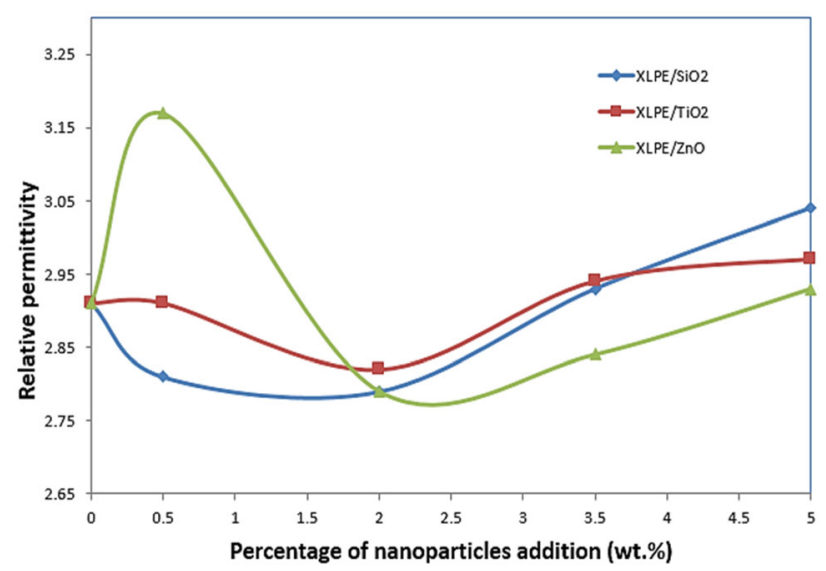

Fig. 10 Relative permittivity of functionalized XLPE nanocomposites at power frequency at different loading of $\mathrm{SiO}_{2}$, $\mathrm{TiO}_{2}$, and $\mathrm{ZnO}$

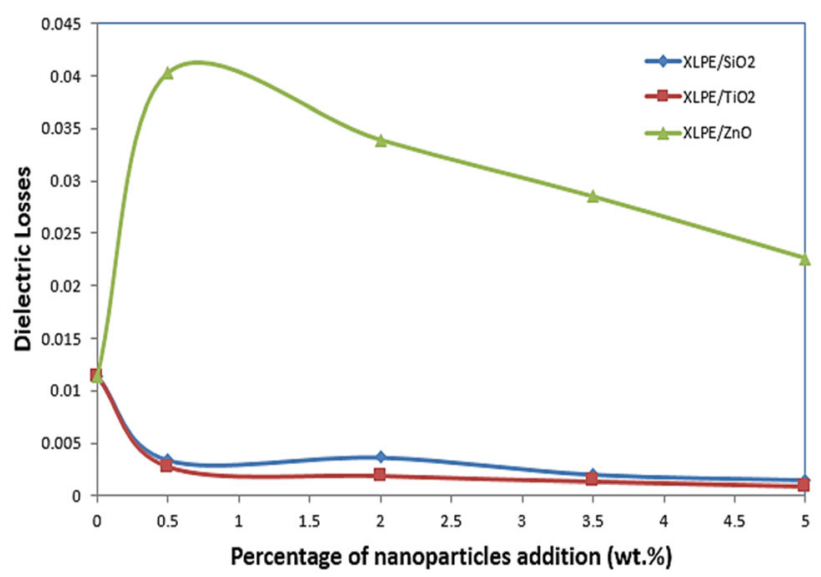

Fig. 11 Dielectric losses of functionalized XLPE nanocomposites at power frequency at different loading of $\mathrm{SiO}_{2}$, $\mathrm{TiO}_{2}$, and $\mathrm{ZnO}$ 


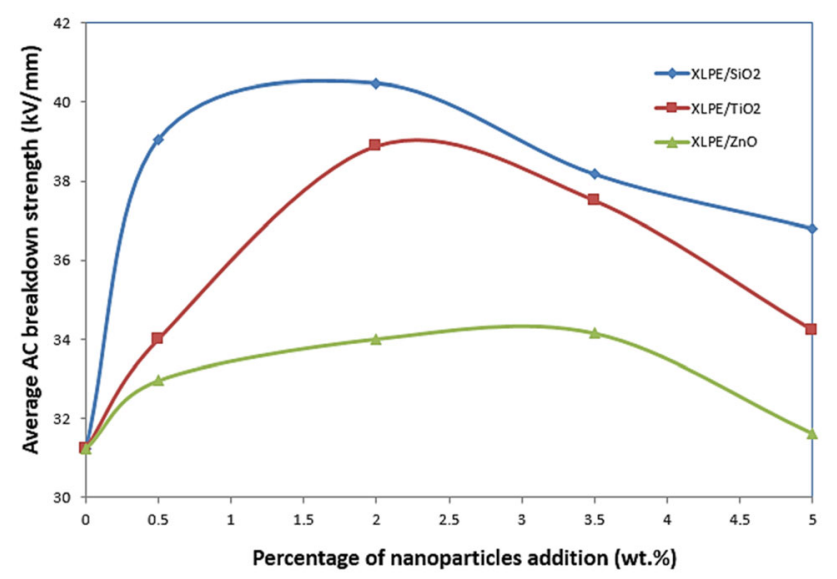

Fig. 12 Average AC breakdown voltage of functionalized XLPE nano-composites at different loading of $\mathrm{SiO}_{2}, \mathrm{TiO}_{2}$, and $\mathrm{ZnO}$

can be seen that the AC BDV is higher in the case of XLPE/amino functionalized NPs samples. This is owing to the functionalized samples' substantial interfacial area between NPs and polymer chains. Furthermore, there is effective NPs dispersion inside the matrix. The AC BDV of the XLPE/ $\mathrm{SiO}_{2}(2 \mathrm{wt} \%$ ) sample increased by $29.6 \%$, whereas the AC BDV of the $\mathrm{XLPE} / \mathrm{SiO}_{2}(0.5 \mathrm{wt} \%)$ sample increased by $25.08 \%$. It can be shown that using silica NPs results in the greatest enhancement and highest AC BDV values.

\section{Conclusions}

The industrial scale of XLPE/nano-composites with different loading ratios of $\mathrm{SiO}_{2}, \mathrm{TiO}_{2}$, and $\mathrm{ZnO} \mathrm{NPs}$ was synthesized and developed based on melt blending method. Surface functionalization of NPs is also discussed as a method for increasing NPs compatibility with the host polymer matrix. The AC BDV, relative permittivity and dielectric losses of the nanocomposites were measured, as well as their thermal, mechanical, and dielectric properties. The incorporation of NPs into the XLPE matrix improved various electrical, thermal, and mechanical properties of the XLPE polymer, particularly silica NPs, and the functionalization NPs resulted in excellent dispersion within the polymer matrix, according to the findings. For relative permittivity, it might be increased or decreased depending on the type, weight fraction, and the size of the filled NPs. On the other hand, in most cases, the dielectric dissipation factor decreased with incorporation of NPs due to the constraint of the charge carrier's movement in nano-composites. The size, type, and weight fraction of NPs all contributed to an improvement in the measured AC BDV of XLPE polymer nano-composites.

\section{Author contributions}

All authors have helped in experiments and improving the overall quality of the manuscript.

\section{Funding}

Open access funding provided by The Science, Technology \& Innovation Funding Authority (STDF) in cooperation with The Egyptian Knowledge Bank (EKB).

\section{Data availability}

All data available.

\section{Declarations}

Conflict of interest The authors declare that they have no conflict of interest.

Open Access This article is licensed under a Creative Commons Attribution 4.0 International License, which permits use, sharing, adaptation, distribution and reproduction in any medium or format, as long as you give appropriate credit to the original author(s) and the source, provide a link to the Creative Commons licence, and indicate if changes were made. The images or other third party material in this article are included in the article's Creative Commons licence, unless indicated otherwise in a credit line to the material. If material is not included in the article's Creative Commons licence and your intended use is not permitted by statutory regulation or exceeds the permitted use, you will need to obtain permission directly from the copyright holder. To view a copy of this licence, visit http://creativecommons.org/licen ses/by/4.0/. 


\section{References}

1. A.N. Ramani et al., The effects of nano fillers on space charge distribution in cross-linked polyethylene. Int. J. Electr. Comput. Eng. 7(6), 3147-3152 (2017)

2. A. Thabet, Effect of nanoparticles on water treeing characteristics in XLPE industrial insulating materials. J. Eng. Sci. 40(1), 191-208 (2012)

3. N.M.K. Abdel-Gawad et al., Enhancement of dielectric and mechanical properties of polyvinyl chloride nanocomposites using functionalized $\mathrm{TiO}_{2}$ nanoparticles. IEEE Trans. Dielectr. Electr. Insul. 24(6), 3490-3499 (2017)

4. N.M.K. Abdel-Gawad et al., Development of industrial scale PVC nanocomposites with comprehensive enhancement in dielectric properties. IET Sci. Meas. Technol. 13(1), 90-96 (2019)

5. Q. Yu et al., Properties of water tree growing in XLPE and composites, in Proceedings of the 2nd international conference on electrical materials and power equipment (ICEMPE). (IEEE, Piscataway, 2019), pp. 409-412

6. S.H. Salh, D.A. Raswl, Thermal stability of polymer composite films based on polyvinyl alcohol doped with different fillers. J. Phys. 2, 5-10 (2018)

7. A.H. Awad et al., A study of some thermal and mechanical properties of HDPE blend with marble and granite dust. Ain Shams Eng. J. 10(2), 353-358 (2019)

8. M. Giżyński, B. Romelczyk-Baishya, Investigation of carbon fiber-reinforced thermoplastic polymers using thermogravimetric analysis. J. Thermoplast. Compos. Mater. 34(1), 126-140 (2021)

9. C. Zhang et al., Improved direct current electrical properties of crosslinked polyethylene modified with the polar group compound. Polymers 11(10), 1624 (2019)

10. S.H. Liu et al., Improving thermal stability of polyurethane through the addition of hyperbranched polysiloxane. Polymers 11(4), 697 (2019)

11. Z. Liu et al., High thermal conductivity of flake graphite reinforced polyethylene composites fabricated by the powder mixing method and the melt-extruding process. Polymers 10(7), 693 (2018)

12. X. Chi et al., Characterization of polypropylene modified by blending elastomer and nano-silica. Materials 11(8), 1321 (2018)

13. J.P. Jose, S. Thomas, Alumina-clay nanoscale hybrid filler assembling in cross-linked polyethylene based nanocomposites: mechanics and thermal properties. Phys. Chem. Chem. Phys. 16(28), 14730-14740 (2014)

14. M. Roy et al., Polymer nanocomposite dielectrics-the role of the interface. IEEE Trans. Dielectr. Electr. Insul. 12(4), 629-643 (2005)

15. A. Said, Z. Anane, Corona lightning overvoltage analysis for a $500 \mathrm{kV}$ hybrid line. IET Gener. Transm. Distrib. 14(4), 532-541 (2019)

16. ASTM D149-09, Standard test method for dielectric breakdown voltage and dielectric strength of solid electrical insulating materials at commercial power frequencies (ASTM, West Conshohocken, 2009)

17. A.R. Said et al., Enhancing electrical, thermal, and mechanical properties of $\mathrm{HV}$ cross-linked polyethylene insulation using silica nanofillers. J. Mater. Eng. Perform. 30(3), 1796-1807 (2021)

18. A.E. Elsayed et al., High voltage cross-linked polyethylene insulator characteristics improvement using functionalized $\mathrm{ZnO}$ nanoparticles. Egypt. J. Chem. 63(12), 4929-4939 (2020)

19. A. Said et al., Enhancing the high voltage XLPE cable insulation characteristics using functionalized $\mathrm{TiO}_{2}$ nanoparticles. Am. J. Polym. Sci. Technol. 6(3), 21-31 (2020)

20. A. Hedir, M. Moudoud, O. Lamrous, S. Rondot, O. Jbara, P. Dony, Ultraviolet radiation aging impact on physicochemical properties of crosslinked polyethylene cable insulation. J. Appl. Polym. Sci. 137, 48575 (2020)

21. C. Huang, X. Qian, R. Yang, Thermal conductivity of polymers and polymer nanocomposites. Mater. Sci. Eng. R 132, 1-22 (2018)

22. C.E. Corcione, M. Frigione, Characterization of nanocomposites by thermal analysis. Materials 5, 2960-2980 (2012)

23. O. Nabinejad, D. Sujan, M.E. Rahman, I.J. Davies, Determination of filler content for natural filler polymer composite by thermogravimetric analysis. J. Therm. Anal. Calorim. 122, 227-233 (2015)

24. Q. Yu, A.P.S. Selvadurai, Mechanical behaviour of a plasticized PVC subjected to ethanol exposure. Polym. Degrad. Stab. 89(1), 109-124 (2005)

Publisher's Note Springer Nature remains neutral with regard to jurisdictional claims in published maps and institutional affiliations. 